\section{Desempeño y competencias directivas en el Autotransporte Público de Carga en Seco. Una revisión empírica en el centro de México}

Performance and managerial competencies in the Public Dry Cargo Autotransport. An empirical review in the middle of Mexico

Eduardo Cruz Aldana; Luis Arturo Guerrero Azpeitia

\section{RESUMEN}

Hoy en día en México el sector de Autotransporte Público de Carga en Seco presenta una dependencia de transportación en carreteras federales, así también muestra la necesidad de optimizar la cadena de suministro asociada a dicha trasportación, en este sentido, ¿Cuál es la relación que existe entre las competencias directivas y el desempeño de empresas dedicadas al Autotransporte Público de Carga en Seco? Para responder a dicha pregunta, se decidió seleccionar como unidad de análisis la Zona Metropolitana del Estado de Hidalgo. Para tal efecto, se establecieron de las competencias directivas desde diferentes posicionamientos dando como resultado una aproximación desde perspectivas conductuales, aptitudinales, formativas, organizacionales y de liderazgo; metodológicamente, se realizó una delimitación de tres tipos de variables: a) la interacción de las competencias directivas; b) la estandarización y; c) su incidencia en el desempeño de las organizaciones que sustentaron el análisis factorial como principal estrategia analítica. Entre los principales resultados se resalta que no existe una relación entre las competencias directivas y el desempeño esperado en el sector bajo análisis y, partiendo de las necesidades individuales, así como de una referencia psicológica y sociológica, se identificó la necesidad de desarrollar las competencias directivas desde una perspectiva holista más que conductual como muestran los hallazgos en aras de mejorar el desempeño de las organizaciones.

Palabras claves: Estandarización; Competencias Directivas; Desempeño

\section{ABSTRACT}

Today in Mexico, the sector of Public Transportation of Dry Cargo presents a transportation agency on federal highways, thus it also shows the need to optimize the supply chain associated with said transportation, in this sense, what is the relationship between managerial skills and the performance of companies dedicated to Public Dry Cargo Transport? To answer this question, it was decided to select as a unit of analysis the Metropolitan Area of the State of Hidalgo in the middle of Mexico, particularly, fieldwork was carried out in 61 companies in the sector under study. For this purpose, a construction of the managerial competences was carried out from different positions resulting in an approach from behavioral, attitudinal, formative, organizational and leadership perspectives; methodologically, a delimitation of three types of variables was carried out: a) the interaction of managerial competences; (b) standardization and c) its impact on the performance of the organizations that supported factor analysis as the main analytical strategy. Of the main results, it is highlighted that there is no relationship between managerial competencies and expected performance in the sector under analysis and based on individual needs, as well as a psychological and sociological reference, the need to develop managerial competencies from a holistic rather than behavioral perspective was identified, as shown by the findings to improve the performance of organizations.

Keywords: Standardization; Management Competencies; Performance
RELIGACIÓN

REVISTA DE CIENCIAS SOCIALES Y HUMANIDADES JOURNAL OF SOCIAL SCIENCES AND HUMANITIES
REVISTA DE CENCIAS SOCIAIS E HUMANAS

INFORMACIÓN:

http://doi.org/10.46652/rgn.v6izo.869 ISSN $2477-9083$

Vol. 6 No. 30, 2021. e210869 Quito, Ecuador

Enviado: octubre 17, 2021

Aceptado: diciembre 13, 2021

Publicado: diciembre 19, 2021

Publicación Continua

Sección Sur-Sur| Peer Reviewed

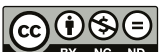

\section{open $\bigcirc$ access}

\section{AUTOR:}

(D) Eduardo Cruz Aldana

Universidad Politécnica Metropolitana de Hidalgo - México

ealdana@upmh.edu.mx

(1) Luis Arturo Guerrero Azpeitia Universidad Politécnica Metropolitana de Hidalgo - México

Iguerrero@upmh.edu.mx

Conflicto de intereses

Los autores declaran que no existe conflicto de interés posible.

Financiamiento

No existió asistencia financiera de partes externas al presente artículo.

Agradecimiento

N/A.

Nota

El presente artículo no se desprende de un trabajo anterior.

ENTIDAD EDITORA 


\section{Introducción}

\subsection{Antecedentes.}

El sector Autotransporte de Público de Carga en Seco (APCS) el cual agrupa las organizaciones que realizan la transportación de mercancías al público en general que lo requiera, por carreteras federales, a través carga en seco (mercancía que no requiere un temperatura o cuidado especializado); a partir del año de 1989, entró en un periodo de la desregulación gubernamental, lo que generó un cambio de paradigma en la forma de administrar dichas organizaciones, debido al crecimiento de las organizaciones de servicio de transportación de mercancía por carretera (Rico, 1998; Jiménez \& Jiménez, 2016).

Cabe mencionar que antes del proceso de desregularización gubernamental del sector APCS, los contratos de transporte y el precio de transportación eran asignados por el gobierno federal, así los directivos de dichas organizaciones no requerían de competencias directivas especializadas en la transportación para poder llevar a cabo la administración de una organización del sector antes comentado (Jiménez, 2018; Cruz et al., 2015; Mercado et al., 2011).

En México, después de la desregularización del sector APCS, en el periodo presidencial de-Carlos Salinas de Gortari, y en específico después de la puesta en marcha del Tratado de Libre Comercio de América del Norte (TLCAN), el desarrollo económico relacionado a la importación de materia prima y exportación de productos ensamblados, permitió aumentar el volumen transportado (materia prima, productos semi-ensamblados y productos finales), dentro de la cadena de suministro, originando la necesidad de mejorar el control de mercancía en tránsito, reduciendo de la incertidumbre y las entregas en tiempo y forma (Rico, 1998).

Sin embargo, el incremento en la oferta (número de organizaciones que prestan el servicio de trasportación) fue mayor que el de la demanda, dando como resultado una disminución o “canibalización” en los precios de este servicio (Mercado et al., 2011). Otro factor relevante fue el crecimiento de las empresas denominadas “Hombres-Camión” haciendo referencia a que son organizaciones que cuentan de 1 a 5 camiones (Morales, 2016) debido a que proliferaron hasta llegar hoy en día a más del 80\%. “Atomizando" el mercado de forma que persiste la administración familiar no profesionalizada y desarrollada de forma empírica.

Así el sector APCS presenta una característica ambigua, debido a que por un lado México tiene una dependencia para la transportación de mercancía en carretera (80\% de su carga) y al mismo tiempo la administración de las empresas de este sector está sujeto a viejas costumbres de dirección, con graves problemas originados por la ineficiencia en la planeación de operaciones, falta de un proceso de capacitación, mala calidad de su servicio y la falta planeación logística, originando una pérdida de competitividad (Mercado et al., 2011; Ortega et al., 2015; Cruz et al., 2018).

Sin embargo, la productividad en México en el año 2021 de acuerdo con The International Institute for Management Development (IMD) publicado por México competitivo (2021) lo sitúa en el lugar número 55 de 64 países, con una calificación de 48.6 (rango emitido de o a 100), siendo su principal fortaleza el tamaño de su mercado; en términos de producción, comercio y población y, dentro de los rubros más bajos se encuentra el de la educación y capacidades con 57.9. Lo 
anterior permite observar una mayor presión a los mandos directivos en relación con un control en los estándares en sus procesos administrativos, a través de las normativas vigentes en México (ver Tabla 1).

Tabla 1. Normativas vigentes del sector APCS en México.

\begin{tabular}{|c|c|}
\hline Normativas vigentes en México. & $\begin{array}{c}\text { Relacionados el cruce fronterizo a Estados } \\
\text { Unidos de América (EUA). }\end{array}$ \\
\hline $\begin{array}{c}\text { ISO 31000:2009-herramienta para evaluar la gestión } \\
\text { de riesgos. }\end{array}$ & $\begin{array}{c}\text { C-TPAT (Customs Trade Partnership Against } \\
\text { Terrorism) }\end{array}$ \\
\hline $\begin{array}{c}\text { ISO 28000:2009- la seguridad de la cadena de } \\
\text { suministros. }\end{array}$ & FAST (Free and Secure Trade) \\
\hline ISO 39001 norma de seguridad vial para las empresas. & BASC (Business Alliance for Secure Commercial) \\
\hline $\begin{array}{c}\text { ISO/IEC 17020:2012-que evalúan la conformidad en } \\
\text { la aplicación de la NOM-061-SCT-2-2000 para la } \\
\text { regulación de las condiciones físico-mecánicas de las } \\
\text { unidades de transporte -, la NOM-012-SCT-2-2008- } \\
\text { que regula el peso y dimensiones de las unidades de } \\
\text { transporte. }\end{array}$ & \\
\hline
\end{tabular}

Fuente: Elaboración propia a partir de Cruz et al., (2018).

Dicha dicotomía se entiende, que, aunque en el contexto del sector APCS existen programas de capacitación y certificación a directivos, hoy en día, en el caso mexicano no muestran un efecto positivo en una mejora en la productividad de dicho sector, y sin embargo, la dependencia de transportación de dicha mercancía en autotransporte de carga en carreteras federales, la cual es mayor al 80\%, muestra la necesidad de optimizar los costos de trasportación para mejor la eficiencia en los rubros de la cadena de suministro sujetas a la necesidad de dicha transportación.

Así, se planteó como pregunta de investigación: ¿Cuál es la relación que existe entre las competencias directivas y el desempeño de empresas dedicadas al Autotransporte Público de Carga en Seco? Para responder a dicha pregunta, se decidió seleccionar como unidad de análisis la Zona Metropolitana del Estado de Hidalgo en tanto zona que presenta un mayor dinamismo económico en la entidad federativa.

\subsection{Análisis teórico de las competencias y de sus sistemas.}

Las competencias directivas están sujetas a la perspectiva conductual, relacionada al conocimiento y a las habilidades directivas que, al controlarlas, el directivo favorece el desempeño esperado por las mismas necesidades de la organización (Taylor, 1967 y Jensen, 2000).

Sin embargo, las habilidades son congruentes a la resolución de problemas y están relacionadas tanto con el conocimiento que se tenga como con la forma de aplicar dicho conocimiento en la administración de los recursos de la organización a fin de que dichos recursos cumplan de forma efectiva las expectativas de la organización, vinculando el conocimiento, habilidades y conductas, el "saber hacer", el "conocer" y otras características individuales que son definidas como el "ser" (Quinn,1990; Olabarrieta, 1998).

Así, para Whetten y Cameron (2005) un directivo debe tener habilidades conductuales, las cuales deberán estar estructuradas en acciones planeadas al momento de proveer solución a un determinado problema con el fin de desarrollar competencias directivas bajo tres categorías: 
- Personales: Orientadas al autoconocimiento, manejo del estrés, solución analítica y creativa de problemas.

- Habilidades interpersonales: Comunicación de apoyo, poder e influencia, motivación de los empleados y manejo de conflictos.

- Grupales: Facultamiento y delegación, formación de equipos eficaces, dirección hacia cambios positivos.

Al llevar a la práctica un proceso vinculante de las competencias directivas con relación al desempeño organizacional bajo la orientación referente a la formación de los directivos, se observa en el "enfoque anglosajón” el cual se orienta a la necesidad de estandarizar los métodos de trabajo a fin de satisfacer las necesidades explícitas e implícitas de sus clientes a través de un énfasis a las competencias genéricas y reconociendo las competencias específicas (McClelland, 1973; Hammel, 1995; Prahalad y Bettis, 1995; Boyatzis, 2002). Por otro lado, el "enfoque francés" centra su trabajo en la persona, vinculando las experiencias y el conocimiento, como un compendio de competencias creadas en la persona, y que a su vez fomenta el desarrollo organizacional (Levy-Leboyer, 1997).

No obstante, al diferenciar las aplicaciones del concepto de competencias con relación a su la definición, operación e implementación, se establece un conjunto de actividades encaminadas a la formación del personal a través de: a) la instalación de dichas competencias; b) denominar y definir el desempeño deseado en cada trabajador; c) delimitar la contribución de cada puesto de trabajo al logro de los objetivos d) enfatizar la gestión del personal a través del desarrollo profesional del individuo. Para lograr lo anterior, se establecen cinco dimensiones de acuerdo con cruz et al., (2018):

- Liderazgo: Proceso vinculante orientado al alineamiento e identificación del conjunto de competencias directivas con relación al desempeño requerido que apoye al logro la realización de la visión organizacional.

- Diagnóstico de competencias: identifica al grupo de competencias directivas con relación a los desempeños esperados, con el fin de ayudar a realizar de mejor forma sus tareas, por medio de los siguientes apartados: caracterización de la actividad o grupo de actividades de trabajo, establecer un fin al desempeñar una actividad o grupo de actividades de trabajo y delimitar las funciones del área ocupacional o ámbito de trabajo.

- Instalación de competencias: integra a las necesidades individuales del directivo con relación al desempeño esperado, a través de la estandarización y la normalización de las competencias exitosas, por medio de los siguientes apartados: a) identificar un grupo de competencias afines al desarrollo profesional determinado; b) establecer un sistema normalizado para un procedimiento de estandarización ligado a una futura institucionalización; c) establecer una norma o un grupo de ellas que sean un referente válido para las instituciones que la utilizarán, los trabajadores y los empleadores y, finalmente; d) establecer normativas en las competencias, y convertirlas en un estándar al nivel en que se haya acordado.

- Desarrollo de competencias: da seguimiento a los esfuerzos y a las acciones encaminadas al logro desempeños establecidos en la fase anterior, a través de la implementación de tácticas secuenciales y orientadas a resultados, por medio de los 
siguientes apartados: a) analizar la descripción de la competencia y su normalización; b) propiciar una formación orientada a generar competencia con referentes claros en normas existentes (tendrá mucha más eficiencia e impacto que aquella desvinculada de las necesidades del sector empresarial).

- Potencialización de competencias: permite generar reacondicionamientos sistémicos a través de cambios planeados, identificando los cambios externos e internos originados por el medio que rodea a la organización, consolidando así el proceso de estandarización de competencias.

Lo anterior permite evidenciar que la gestión del desarrollo de competencias directivas es un recurso vinculado a la tecnoestructura organizacional, debido a que tiene como fin mejorar la eficacia de sus resultados, haciendo frente a problemáticas cotidianas tales como: factores originados por las amenazas de seguridad, entornos globalizados y cambios climáticos entre otros (Mintzberg, 1973; Redwood et al., 1999; Blunt et al., 2014).

Sin embargo, dentro del análisis contextual realizado por Cruz et al. (2018) se observó que en las etapas de las cinco dimensiones de un sistema de competencias, bajo un análisis de contexto de los principales modelos de capacitación de competencias directivas del APCS (definidos como: el American Production and Inventory Control Society (APICS); Human Resources Professionals Association; Comisión Sistema Nacional de Certificación de Competencias Laborales (Chilevalora); el Inside Careers; Clasificación Brasileña de Ocupaciones (CBO) y el Sistema Nacional de Competencias (SNC)), solo cuatro dimensiones son aplicadas, así la última dimensión referida a "la potencialización" no es aplicada en ninguno de los modelos comentados.

Lo anterior permite cuestionar si el accionar de la competencia directiva para crear y utilizar sus recursos al dar solución a uno o un conjunto de problemas, tomando en cuenta el significado de desempeño, es decir, implementarlo en situaciones reales. Por ello es importante delimitar que el presente estudio se basa también en un principio de alineamiento estratégico de una organización, al medir su desempeño estructurado en el cuadro de mando integral, el cual delimita dicha medición de del desempeño organizacional en cuarto perspectivas de acuerdo con Kaplan y Norton (2001).

Financiera: representa la medida en que se ejecuta la estrategia de las organizaciones del APCS, con relación a los temas de administración de ingresos, reducciones de costo, aumento de la productividad y de utilización de los activos, se retomaron las necesidades expuestas en el análisis de los modelos de competencias relacionadas a aspectos financieros y se definieron las siguientes tareas y demandas de cargos directivos (Kaplan y Norton, 2001).

La satisfacción y retención de los clientes: está orientada hacia la identificación de los clientes, la proporción de valor de servicio que se ofrece, satisfacción del cliente, fidelidad del cliente, cuota de mercado y adquisición de clientes, en el sector APCS (Tracy y Wierseme, 1995; Porter, 1999; Kaplan y Norton, 2001).

Proceso interno: está orientada a satisfacer las expectativas de los clientes y de los dueños del dinero invertido en la organización, así también se entiende que esta perspectiva está orientada a los procesos clave como la integración de aspectos relacionados al servicio 
prestado, con relación al control de combustible, planificación y asignación de rutas, control de costos y tiempos de paro de cada unidad (Tracy y Wierseme, 1995; Porter, 1999; Kaplan y Norton, 2001).

Recurso humano: para esta investigación, se hace referencia a la toma de decisiones directivas del APCS, con relación a las acciones enfocadas al ambiente de competencia requeridos para que las organizaciones de APCS mejoren de forma continua el servicio prestado bajo tres enfoques: la satisfacción, retención y productividad del trabajador (Kaplan y Norton, 2001; Amaratunga et al., 2001).

Con relación a la perspectiva de liderazgo, para esta investigación se hace referencia a la influencia que el directivo de APCS y que de acuerdo con Schein (1978) está enfocado a la forma en la cual un líder debe manejar a sus subordinados. Se define que el liderazgo coadyuva a desarrollar una cultura organizacional a través de orientar las acciones de los colaboradores a resultados alineados a la estrategia de las organizaciones del APSC (Cameron y Quinn, 1999; Cruz \& Guerrero 2021).

\section{Metodología}

Así la investigación reportada por Cruz et al., (2018) la cual se toma como base en este trabajo, muestra la necesidad desarrollar competencias directivas orientadas al desempeño organizacional con el fin de fortalecer no solo las habilidades y competencias directivas, sino también orientar el desarrollo organizacional con relación al desempeño organizacional a través de cuatro sustentos básico, los cuales son citados de Kaplan y Norton (2001).

En atención a la problemática expuesta, se alude a una falta de competencia directivas; ya que al momento de emitir decisiones en la mayoría de las ocasiones se realizan por experiencias de las personas que dirigen la parte operativa de la organización, observando una falta de conocimientos, habilidades y técnicas; ya que se carece de una herramienta o método que ayuden a mejorar el desempeño del APCS para el cumplimiento de los objetivos de la organización.

Cabe mencionar que se realizó una delimitación a razón de tres tipos de variables de acuerdo con la fundamentación teórica de un MHTE; a) la interacción de las competencias directivas; b) la estandarización y; c) su incidencia en el desempeño de las organizaciones de la ZMEH. La decisión metodológica de lo anterior responde a las recomendaciones de diversos especialistas tales como Bell (1973), Torres \& Navarro (2007), Naghi (2010) y Beckhard (1986) tal como se puede ver en la Tabla 2. 
Tabla 2. Variables del modelo de investigación.

\begin{tabular}{|c|c|c|}
\hline Tipos de Variables. & Características de la variable. & Explicación. \\
\hline $\begin{array}{l}\text { Variable dependiente: } \\
\text { Competencias Directivas }\end{array}$ & $\begin{array}{l}\text { Conocimientos. } \\
\text { Aptitudes y habilidades }\end{array}$ & $\begin{array}{l}\text { Se considera que las competencias } \\
\text { directivas tienen una inferencia positiva con } \\
\text { relación al desempeño. }\end{array}$ \\
\hline $\begin{array}{l}\text { Variable independiente: } \\
\text { Desempeño }\end{array}$ & $\begin{array}{l}\text { Procesos financieros. } \\
\text { Orientación hacia el cliente. } \\
\text { Procesos internos. } \\
\text { Recursos Humanos. } \\
\text { Liderazgo. }\end{array}$ & $\begin{array}{l}\text { Se considera que el desempeño genera } \\
\text { causas y condiciones a las competencias } \\
\text { directivas. }\end{array}$ \\
\hline $\begin{array}{l}\text { Variable interviniente: } \\
\text { Estandarización }\end{array}$ & $\begin{array}{l}\text { Identificación de competencias. } \\
\text { Normalización de competencias. } \\
\text { Formación basada en } \\
\text { competencias. } \\
\text { Certificación de competencias. }\end{array}$ & $\begin{array}{l}\text { Se considera que la estandarización influye } \\
\text { en la relación: competencias directivas } \\
\text { y el desempeño, siendo una variable } \\
\text { interviniente entre ellas. }\end{array}$ \\
\hline
\end{tabular}

Fuente: Adaptado de Hernández et al., (2005); Torres y Navarro (2007) \& Naghi (2010).

Con base en lo anterior, se proponen las siguientes variables, las cuales se consideran como competencias directivas y desempeño.

X: Competencias directivas.

X1: Competencias directivas financieras.

X2: Competencias directivas de orientación al cliente.

$\mathrm{X}_{3}$ : Competencias directivas de proceso interno.

$\mathrm{X}_{4}$ : Competencias directivas de recursos humanos.

$X_{5}$ : Competencias directivas de liderazgo.

Y: Desempeño.

Y1: Desempeño directivo financieros.

Y2: Desempeño directivo de orientación al cliente.

Y3: Desempeño directivo de proceso interno.

Y4: Desempeño directivo de recursos humanos.

Y5: Desempeño directivo de liderazgo.

La hipótesis de esta investigación establece que existe interacción entre las competencias directivas $(X)$ y el desempeño $(Y)$, a través de los procesos de estandarización, en el sector de Autotransporte Público de Carga en Seco, de la zona metropolitana del estado de Hidalgo, en tanto que las hipótesis de trabajo se pueden observar en la Tabla 3. 
Tabla 3. Hipótesis de trabajo.

\begin{tabular}{|c|c|}
\hline$H X_{1}-Y_{1}$ & Las Competencias directivas financieras tienen relación con el desempeño directivo financieros. \\
\hline$H X_{2}-Y_{1}$ & Las competencias directivas de orientación al cliente tienen relación con el desempeño directivo financiero. \\
\hline $\mathrm{HX}_{3}-\mathrm{Y}_{1}$ & Las Competencias directivas de proceso interno tienen relación con el desempeño directivo financiero. \\
\hline $\mathrm{HX}_{4}-\mathrm{Y}_{1}$ & Las competencias directivas de recursos humanos tienen relación con el desempeño directivo financiero. \\
\hline $\mathrm{HX}_{5}-\mathrm{Y}_{1}$ & Las Competencias directivas de liderazgo tienen relación con el desempeño directivo financiero. \\
\hline$H X_{1}-Y_{2}$ & Las Competencias directivas financieras tienen relación con el desempeño directivo de orientación al cliente. \\
\hline $\mathrm{HX}_{2}-\mathrm{Y}_{2}:$ & $\begin{array}{l}\text { Competencias directivas de orientación al cliente tienen relación con desempeño directivo de orientación al } \\
\text { cliente. }\end{array}$ \\
\hline $\mathrm{H}_{3}-\mathrm{Y}_{2}:$ & Competencias directivas de proceso interno tienen relación con desempeño directivo de orientación al cliente. \\
\hline $\mathrm{HX}_{4}-\mathrm{Y}_{2}:$ & Competencias directivas de recursos humanos tienen relación con el desempeño directivo de orientación al cliente. \\
\hline $\mathrm{HX}_{5}-\mathrm{Y}_{2}:$ & Las Competencias directivas de liderazgo tienen relación con desempeño directivo de orientación al cliente. \\
\hline$H X_{1}-Y_{3}:$ & Las Competencias directivas financieras tienen relación con el desempeño directivo de proceso interno. \\
\hline $\mathrm{HX}_{2}-\mathrm{Y}_{3}:$ & Competencias directivas de orientación al cliente tienen relación con el desempeño directivo de proceso interno \\
\hline $\mathrm{H}_{3} \mathrm{Y}_{3}$ & Competencias directivas de proceso interno tienen relación con el desempeño directivo de proceso interno. \\
\hline $\mathrm{HX}_{4}-\mathrm{Y}_{3}$ & Las Competencias directivas de recursos humanos tienen relación con el desempeño directivo de proceso interno. \\
\hline $\mathrm{H}_{5}-\mathrm{Y}_{3}:$ & Las Competencias directivas de liderazgo tienen relación con el desempeño directivo de proceso interno. \\
\hline $\mathrm{HX}_{1}-\mathrm{Y}_{4}:$ & Las Competencias directivas financieras tienen relación con el Desempeño directivo de recursos humanos. \\
\hline $\mathrm{HX}_{2}-\mathrm{Y}_{4}:$ & Competencias directivas de orientación al cliente tienen relación con el Desempeño directivo de recursos humanos. \\
\hline $\mathrm{H}_{3}-\mathrm{Y}_{4}:$ & Competencias directivas de proceso interno tienen relación con el Desempeño directivo de recursos humanos. \\
\hline $\mathrm{HX}_{4}-\mathrm{Y}_{4}:$ & $\begin{array}{l}\text { Las Competencias directivas de recursos humanos tienen relación con el Desempeño directivo de recursos } \\
\text { humanos. }\end{array}$ \\
\hline $\mathrm{H}_{5}-\mathrm{Y}_{4}:$ & Las Competencias directivas de liderazgo tienen relación con el Desempeño directivo de recursos humanos. \\
\hline $\mathrm{HX}_{1}-\mathrm{Y}_{5}:$ & Las Competencias directivas financieras tienen relación con el desempeño directivo de liderazgo. \\
\hline $\mathrm{HX}_{2}-\mathrm{Y}_{5}$ & Competencias directivas de orientación al cliente tienen relación con el desempeño directivo de liderazgo. \\
\hline $\mathrm{H}_{3}-\mathrm{Y}_{5}:$ & Competencias directivas de proceso interno tienen relación con el Desempeño directivo de recursos humanos. \\
\hline $\mathrm{H}_{4}-\mathrm{Y}_{5}:$ & $\begin{array}{l}\text { Las Competencias directivas de recursos humanos tienen relación con el Desempeño directivo de recursos } \\
\text { humanos }\end{array}$ \\
\hline $\mathrm{H} \mathrm{S}_{5}-\mathrm{Y}_{5}$ & ncias directivas de liderazgo tienen relación con el desempeño directivo de liderazgo. \\
\hline
\end{tabular}

\section{Fuente: Elaboración propia.}

\section{Selección de la población.}

Para Tovar (2011), desde 1976 en el estado de Hidalgo, las zonas metropolitanas de Pachuca, Tulancingo y Tula de Allende se han desarrollado a razón de la industrialización de los estados limítrofes, y esto ha originado el que se modifiquen las normativas y las actividades relacionadas con la transportación de mercancías, como se puede ver en la Figura 1. 
Figura 1. Zona Metropolitana del estado de Hidalgo.

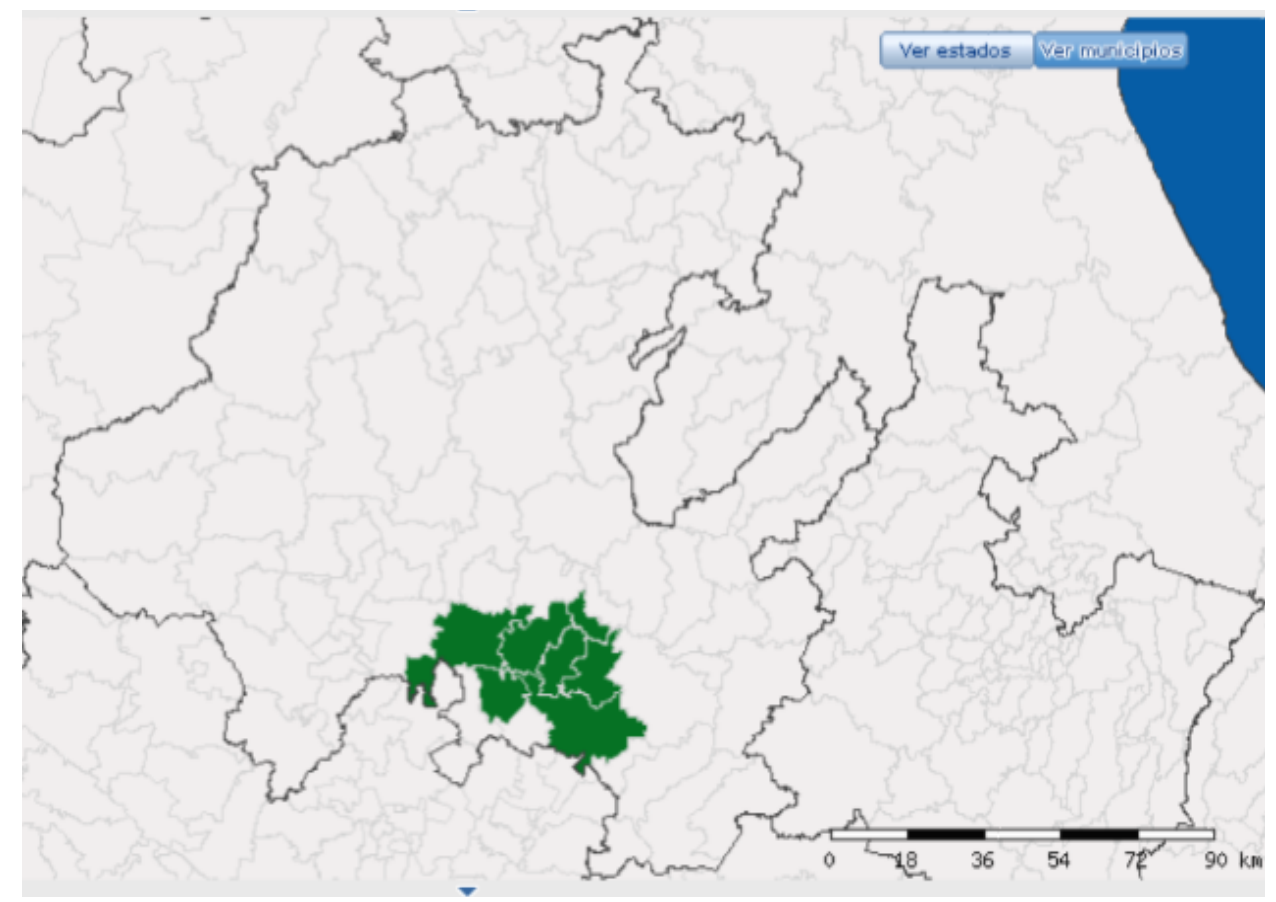

Fuente: Adaptado de Tovar (2011).

Este proyecto está situado en las zonas metropolitanas de Pachuca de Soto, Tulancingo y Tula, las cuales conforman a su vez la Zona Metropolitana del Estado de Hidalgo (ZMEH), con una temporalidad 2018 y bajo un análisis de corte cuantitativo. Las unidades de análisis seleccionadas fueron 61 empresas del sector APCS y la obtención de los datos fue a través de entrevistas estructuradas realizadas a sus directivos, como se puede ver en la Tabla 4.

Tabla 4. Municipios de la zona metropolitana del estado de Hidalgo.

\begin{tabular}{ccc}
\hline Zona metropolitana de Pachuca & $\begin{array}{c}\text { Zona metropolitana de } \\
\text { Tulancingo. }\end{array}$ & Zona metropolitana de Tula. \\
\hline Pachuca de Soto & Tulancingo de Bravo, \\
Mineral de la Reforma & $\begin{array}{c}\text { Cuautepec de Hinojosa } \\
\text { Santiago Tulantepec de Lugo } \\
\text { Guerrero }\end{array}$ & Tula de Allende \\
Epazoyucan & Atitalaquia \\
Mineral del Monte & Atotonilco de Tula \\
San Agustín Tlaxiaca & Tlahuelilpan y \\
Zapotlán de Juárez & Tlaxcoapan \\
Zempoala & Tepeji del Rio \\
\hline
\end{tabular}

Fuente: Adaptado de Herrera et al., (2005).

Las empresas del sector APCS se obtuvieron mediante la consulta a la base de datos de INEGI (2018), y se pueden observar un total de 61 empresas distribuidas en los municipios que se componen de acuerdo con Tovar (2011) en la zona metropolitana de estado de Hidalgo (Ver Tabla 5). 
Tabla 5. Número de empresas por municipio en la ZMEH.

\begin{tabular}{|l|c|}
\hline Municipio & Número Empresas \\
\hline Tulancingo de Bravo & 19 \\
\hline Pachuca de Soto & 15 \\
\hline Tizayuca & 6 \\
\hline Atotonilco de Tula & 6 \\
\hline Tepeji del río de Ocampo & 5 \\
\hline Tula de Allende & 4 \\
\hline Mineral de la Reforma & 3 \\
\hline Tlahuelilpan & 3 \\
\hline Atitalaquia & 1 \\
\hline
\end{tabular}

Fuente: Adaptado de INEGI (2018).

Los elementos de análisis en esta investigación fueron las competencias de las directivas y su correspondencia con el desempeño de dichas organizaciones. Por ello, se seleccionaron del Instituto Nacional de Estadística, Geografía e Informática de México (INEGI) en tono a la transportación carretera, transportación publica, transportación de mercancía en seco, el método de evaluación se estableció a través de 3 rubros:

Nivel básico: Hace referencia a las competencias mínimas, denominadas "básicas", dentro del cual solo se transporta la mercancía de un punto a otro, bajo contrato o por consignación, estableciendo conocimiento en precios, tiempo promedio, variación de tiempo de tránsito, pedidos y daños. Nivel medio: Como un segundo grado de desarrollo se identificaron, no solo el dominio de los de nivel básico, sino la estandarización de sus procesos, el alineamiento estratégico, integración del uso de tecnología y la orientación a hacia sus clientes. Nivel alto: Tercer grado de desarrollo, se identificaron aunados a los elementos previstos en los niveles básico y medio, elementos de intermodalidad, certificaciones y acreditaciones con distintos entes que permitan ampliar la penetración a distintos mercados nacionales e internacionales (Cruz et al., 2018).

Así se construyeron una serie de cuestionamientos, agrupados al tipo de desempeño a evaluar, como también las competencias relacionadas a cada desempeño, definiendo un grupo de peguntas y opciones de respuesta que permitieron identificar las posturas del directivo antes comentadas en cada una de las tres apreciaciones, y se dejó una cuarta alternativa abierta para que anotara o comentara si su accionar en la empresa es distinta a cada una de las observaciones ofrecidas. Dichos cuestionamientos fueron revisados a través de un conjunto expertos del área de Ingeniería Industrial por parte de la Universidad Autónoma del Estado de Hidalgo (UAEH), del área de Ingeniería en Logística y Transporte por parte de la Universidad Politécnica Metropolitana de Hidalgo (UPMH), así también el Comité Científico del Congreso Internacional en Logística y Cadena de Suministro (CiLOG).

Una vez que se identificaron las organizaciones a analizar en esta investigación, se realizó el trabajo de campo con el fin de sensibilizar y aplicar las encuestas en cada organización del 
APCS seleccionada. Posteriormente se realizó un estudio factorial, dentro del cual se observó la interacción de las variables: competencias directivas y el desempeño, generando los resultados que a continuación se presentan.

\section{Análisis y discusión de los resultados.}

De acuerdo con los resultados obtenidos mediante el análisis factorial, se formó un modelo estructural de análisis de correlación de Pearson, que permitió observar el comportamiento de cada variable, con el fin de identificar las tendencias empíricas obtenidas de las encuestas recopilados de los directivos del APCS de la ZMEH.

Así se observó que las organizaciones del sector de APCS de la ZMEH poseen una variabilidad (valor probabilístico) de 0.993, estableciendo (2-1) grados de libertad para las competencias directivas y (5-1) grados de libertad para el desempeño, lo cual permitió a través de la tabla F con un alfa 0.05 establecer una variabilidad máxima de 0.06; lo cual mostró que no existe una relación entre las competencias directivas y el desempeño, ver Tabla 6.

Tabla 6. Comparativo entre competencias directivas $(X)$, desempeño (Y) y su interacción.

\begin{tabular}{|l|c|c|c|c|c|}
\hline \multicolumn{1}{|c|}{ Fuente } & $\begin{array}{c}\text { Grados de } \\
\text { libertad }\end{array}$ & $\begin{array}{c}\text { Sumatoria } \\
\text { cuadrática }\end{array}$ & $\begin{array}{c}\text { Media } \\
\text { Cuadrática }\end{array}$ & $\begin{array}{c}\text { Valor } \\
\text { estadístico }\end{array}$ & $\begin{array}{c}\text { Valor } \\
\text { probabilístico }\end{array}$ \\
\hline $\begin{array}{l}\text { Competencias } \\
\text { directivas }\end{array}$ & 1 & 0.00008 & 0.0000830 & 0.00 & 0.966 \\
\hline Desempeño & 4 & 0.16741 & 0.0418536 & 0.04 & 0.447 \\
\hline $\begin{array}{l}\text { Interacción de } \\
\text { ambas variables }\end{array}$ & 4 & 0.01104 & 0.0027599 & 0.06 & 0.993 \\
\hline Error & 90 & 4.02711 & 0.0447456 & & \\
\hline Total & 99 & 4.20564 & & & \\
\hline
\end{tabular}

Fuente: Elaboración propia.

Así el vínculo entre las competencias y el desempeño, en este análisis sustentan la propuesta de creación de un MHTE vincule las necesidades de los clientes (los requerimientos de capacidad, tarifas, cotizaciones, gestión de mercado, movilización de carga, garantías, término de contratos y orden de servicio), con los objetivos, metas y fines organizacionales y, que estén alineados a los rediseños estructurales, desde el contenido de cargas de trabajo, hasta la comunicación, retro evaluación y evaluación misma de sus resultados (desempeño).

Así el directivo, aunque posea un compendio de competencias y sea el portador del pensamiento cognitivo, no estructura las estrategias que le permitan a su equipo alcanzar las metas y objetivos delegados. Prueba de ello se observa al análisis las competencias directivas del APCS de la ZMEH, en donde el constructo de competencias financieras presenta un menor desarrollo que el resto de los agrupamientos de competencias directivas, cómo se puede ver en la Figura 2. 
Figura 2. Media aritmética de las competencias directivas en el sector APCS de la ZMEH.

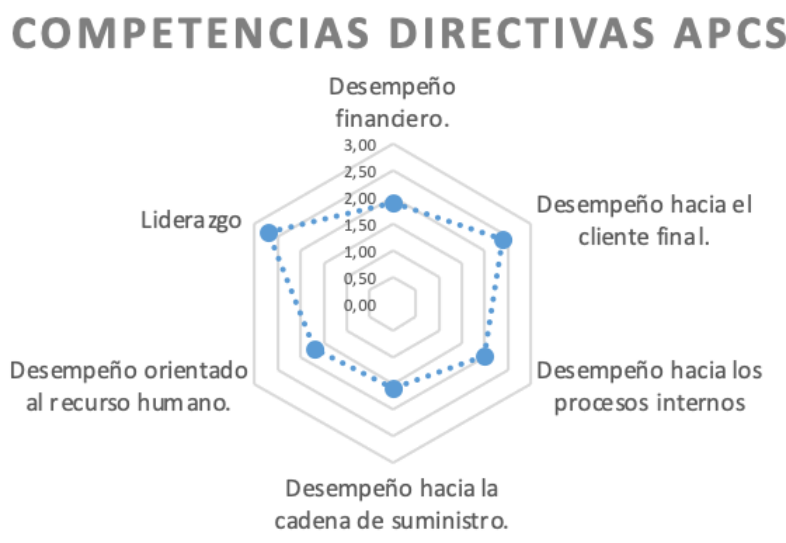

Fuente: Elaboración propia.

También se identificó la misma tendencia en los agrupamientos de desempeño, debido a que presentaron las mismas características que los agrupamientos de competencias directivas, como se puede ver en la Figura 3 en donde los agrupamientos de los desempeños de liderazgo y orientación al cliente posen un desarrollo superior al de desempeño financiero.

Figura 3: Media aritmética del desempeño en el sector APCS de la ZMEH.

\section{DESEMPEÑO APCS}

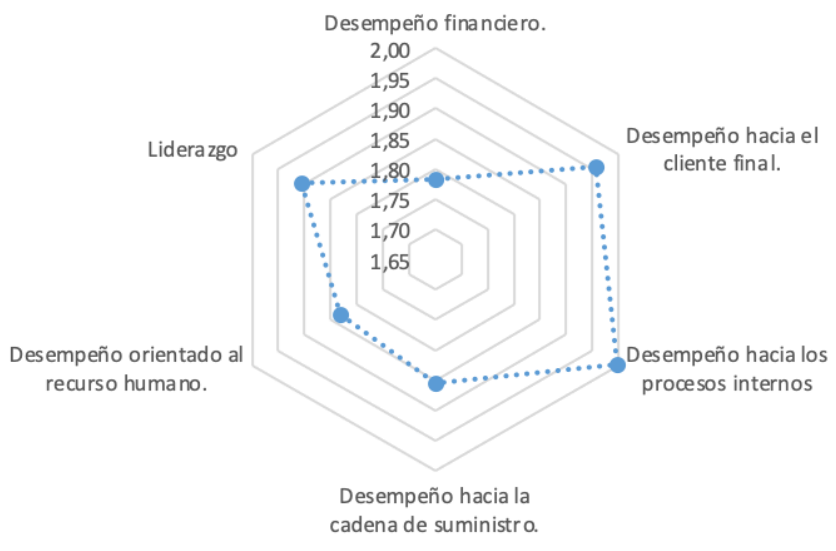

Fuente: Elaboración propia para la presente investigación.

De acuerdo con los resultados obtenidos mediante el análisis factorial, se formó un modelo estructural de análisis de correlación de Pearson, que permitió observar el comportamiento de cada variable, con el fin de identificar las tendencias empíricas obtenidas de las encuestas aplicadas a los directivos del APCS de la ZMEH, ver Figura 4. 
Figura 4. Análisis de correlación Pearson del modelo estructural.

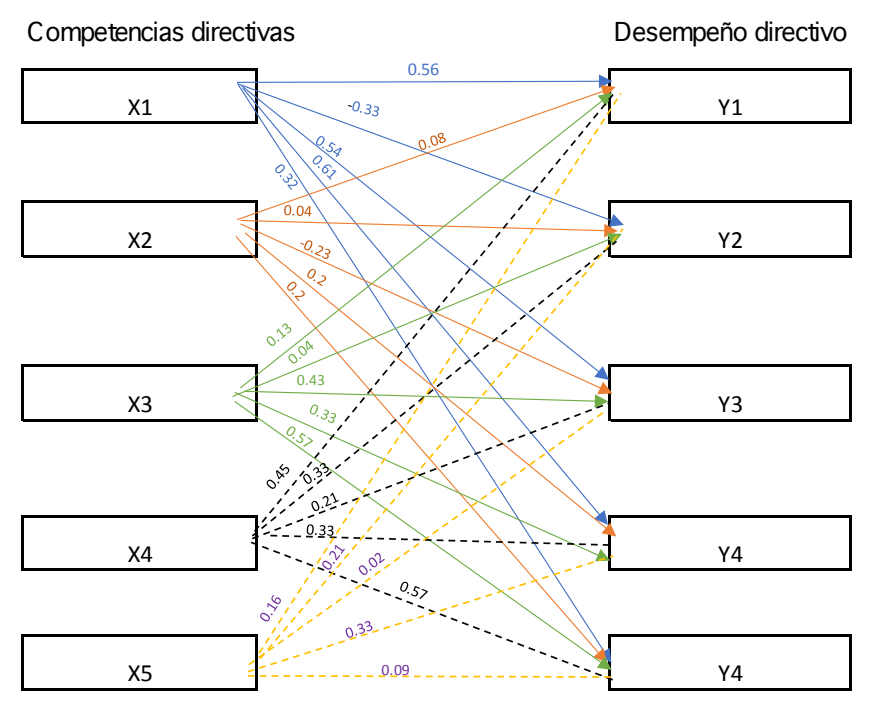

Fuente: Elaboración propia.

También se identificó, que las organizaciones APCS de este estudio, se caracterizaron por entender y aceptar a las competencias directivas. No obstante, se evidencia que el liderazgo es la variable de mayor importancia (1.9529) por lo que, al construir un modelo de competencias directivas se sustenta como el componente primario, que de manera empírica en la correlación de bajamoderada entre las competencias directivas analizadas resalta su importancia (0.423), pues estas carecen de una estructura que permita alinear los esfuerzos organizacionales.

Así también se observó que las competencias directivas analizadas de sector de APCS de la ZMEH, no tienen una inferencia positiva en el desempeño esperado, lo que representa el rechazo a la hipótesis nula de esta investigación. Es decir, que, al analizar la toma de decisiones del directivo a razón de las necesidades individuales de cada uno de ellos, existe priorización a razón de necesidades de cada organización y, esto ocasiona que los desempeños sean distintos (a criterio de cada directivo). Dicho criterio se crea a razón de las necesidades individuales vistas en función de la experiencia, habilidades y bagaje propio del conocimiento relacionado al APCS.

Así el análisis de las dos variables a tratar: competencias directivas (Variable dependiente) y el Desempeño (Variable independiente) permiten aceptar la necesidad de vincular a las competencias directivas y al desempeño en un modelo que estandarice dicha interacción, lo que da sustento y validez empírica a la presente investigación. El análisis de las hipótesis de trabajo se muestra a continuación en la Tabla 7. 
Tabla 7. Matriz de resultados de hipótesis de trabajo.

\begin{tabular}{|c|c|c|c|}
\hline & Relación causal & Estadístico T & Hipótesis \\
\hline $\mathrm{X}_{1-Y_{1}}$ & 0.03 & 0.877 & Aceptada \\
\hline$X_{2}-Y_{1}$ & 0.06 & 0.819 & Aceptada \\
\hline$X_{3}-Y_{1}$ & 0.15 & 0.707 & Aceptada \\
\hline$X_{4}-Y_{1}$ & 2.03 & 0.192 & Rechazada \\
\hline$X_{5}-Y_{1}$ & 0.23 & 0.642 & Aceptada \\
\hline$X_{1}-Y_{2}$ & 1.04 & 0.338 & Rechazada \\
\hline$X_{2}-Y_{2}$ & 0.01 & 0.908 & Aceptada \\
\hline$X_{3}-Y_{2}$ & 1.83 & 0.213 & Rechazada \\
\hline$X_{4}-Y_{2}$ & 1.00 & 0.346 & Rechazada \\
\hline$X_{5}-Y_{2}$ & 0.39 & 0.548 & Aceptada \\
\hline$X_{1}-Y_{3}$ & 3.32 & 0.106 & Rechazada \\
\hline$X_{2}-Y_{3}$ & 0.47 & 0.511 & Aceptada \\
\hline$X_{3}-Y_{3}$ & 0.27 & 0.354 & Aceptada \\
\hline$X_{4}-Y_{3}$ & 0.14 & 0.716 & Aceptada \\
\hline$X_{5}-Y_{3}$ & 0.01 & 0.937 & Aceptada \\
\hline$X_{1-} Y_{4}$ & 4.75 & 0.061 & Rechazada \\
\hline$X_{2}-Y_{4}$ & 0.36 & 0.563 & Aceptada \\
\hline$X_{3}-Y_{4}$ & 3.85 & 0.086 & Rechazada \\
\hline$X_{4}-Y_{4}$ & 0.38 & 0.553 & Aceptada \\
\hline$X_{5}-Y_{4}$ & 0.99 & 0.349 & Rechazada \\
\hline$X_{1}-Y_{5}$ & 0.94 & 0.361 & Rechazada \\
\hline$X_{2}-Y_{5}$ & 0.95 & 0.358 & Rechazada \\
\hline$X_{3}-Y_{5}$ & 0.56 & 0.476 & Rechazada \\
\hline$X_{4}-Y_{5}$ & 0.05 & 0.822 & Aceptada \\
\hline$X_{5}-Y_{5}$ & 0.07 & 0.802 & Aceptada \\
\hline
\end{tabular}

Fuente: Elaboración propia.

De acuerdo con el análisis de las hipótesis de trabajo, se identificó que cada competencia directiva $\left(X_{1}, X_{2}, X_{3}, X_{4}\right.$ y $\left.X_{5}\right)$ muestran una interacción positiva respecto al desempeño generado ( $Y_{1}$, $Y_{2}, Y_{3}, Y_{4}$ y $\left.Y_{5}\right)$. Sin embargo, la interacción de cada competencia directiva $(X)$ a razón de los desempeños distintos a su perspectiva, muestran un resultado heterogéneo, debido a que en la instalación de competencias directivas $(X)$, no planean la interrelación que pudieran tener con las otras perspectivas de desempeño (Y), perdiendo así la visión holística del sistema.

Así también se observó que la competencia directiva de liderazgo ( $\left.X_{5}\right)$ mostró homogeneidad en las cinco perspectivas de desempeño $(Y$ ) (se aprobaron cada una de las hipótesis donde se vinculó a la competencia directivas de liderazgo) siendo esta perspectiva la de mayor interacción con el resto desempeños. Las competencias financieras $\left(\mathrm{X}_{1}\right)$ no mostraron una interacción con 
los desempeños de procesos internos $\left(Y_{2}\right)$, orientación al cliente $\left(Y_{3}\right)$, recursos humanos $\left(Y_{4}\right)$ y liderazgo (Y5); siendo la competencia directiva con menos relación al resto de desempeños, solo mostro interacción con el desempeño financiero $\left(Y_{1}\right)$. La orientación al cliente $\left(X_{2}\right)$ muestra interacción con el desempeño, financiero $\left(Y_{1}\right)$, orientación al cliente $\left(Y_{2}\right)$, proceso interno $\left(Y_{3}\right)$, recurso humano $\left(\mathrm{Y}_{4}\right)$, pero no lo hace con el liderazgo $\left(\mathrm{Y}_{5}\right)$.

Las competencias directivas de proceso interno $\left(X_{3}\right)$ muestran interacción con el desempeño financiero $\left(Y_{1}\right)$ y su propio desempeño $\left(Y_{3}\right)$, pero no lo hace con los desempeños de orientación al cliente $\left(Y_{2}\right)$, recursos humanos $\left(Y_{4}\right)$, ni con el de liderazgo $\left(Y_{5}\right)$. Las competencias de recursos humanos $\left(X_{4}\right)$ muestran interacción con los desempeños de recursos humanos $\left(Y_{4}\right)$, de procesos internos $\left(Y_{3}\right)$, orientación al cliente $\left(Y_{2}\right)$, liderazgo $\left(Y_{5}\right)$, pero no con el desempeño de procesos internos $\left(Y_{2}\right)$.

El análisis de esta investigación establece bajo un enfoque teórico, metodológico y empírico, que las competencias directivas infieren en el desempeño. Sin embargo, dicho análisis funda a la estandarización como una variable interviniente entre las competencias directivas y el desempeño. Para ello se identificó que el liderazgo tanto en la variable de competencias directivas (X5), como en el desempeño (Y5) interviene como el factor ponderarte y vinculante entre las variables de competencias directivas y del desempeño y, que está relación a medida que se estandarice, se fortalece su vínculo.

De este modo, el liderazgo de acuerdo con el análisis teórico y contextual se establece a razón de las habilidades humanas, técnicas y conceptuales, que fijan la orientación del tipo y características de las competencias directivas de acuerdo con el desempeño requerido. Lo anterior permite vincular a las competencias directivas y al desempeño a razón de las variables mostradas $\left(X_{1}, X_{2}\right.$, $X_{3}, X_{4}, X_{5}, Y_{1}, Y_{2}, Y_{3}, Y_{4}, y Y_{5}$.

\section{Conclusiones}

Se reconoce la importancia de evaluar la interacción de las variables analizadas a fin de que no se duplique esfuerzos y que exista correspondencia entre ellas, debido a que en el análisis de las hipótesis de trabajos se observó la ausencia de una planeación holista en las variables antes comentadas. Estas necesidades individuales están basadas a través de los enfoques "Psicológicos" y "Sociológicos", lo que se evidencia al identificar que algunas agrupaciones de competencias directivas enfocadas a desempeños específicos son heterogéneas en su grado de desarrollo. Prueba de ello se observa al analizar el comportamiento de las hipótesis de trabajo en las cuales se muestra un comportamiento heterogéneo en la interrelación de las variables de competencias directivas y de desempeño.

De acuerdo con lo anterior, se entiende que toda organización define sus necesidades acordes a la visión estratégica que tenga de sí misma y, que dicho reposicionamiento implica una caracterización distinta de las necesidades de competencias directivas a desarrollar. Otro factor relevante observado se ve en las necesidades individuales y grupales a razón de los aspectos psicológicos y de la tecnoestructura a razón de los aspectos sociológicos, los cuales permiten al directivo no solo ser poseedor del pensamiento cognitivo, sino conductista y esto permite 
sensibilizar los esfuerzos a organizacionales a un fin común. Así, los cambios generados a partir de las competencias directivas generarán un efecto holista en los desempeños organizacionales.

Se puede observar que dentro del estudio se analizó el comportamiento de la ZMEH, sin embargo, se recomienda para futuros estudios ampliar la zona de influencia de análisis de competencias directivas de APCS a través de organismos como la Cámara Nacional de Autotransporte de Carga (CANACAR), la Asociación Nacional de Transporte Privado (ANTP), la Conferencia Nacional de Transportistas Mexicanos (CONATRAM), la Alianza Mexicana de Organizaciones Transportistas (AMOTAC), con el fin de proporcionar un análisis robusto, a través de la respuesta de sus asociados.

De las recomendaciones para futuras investigación se establece: 1) Analizar los distintos clústeres del APCS en México con el fin de interpretar el comportamiento a razón de la caracterización de los distintos tipos de mercado de autotransporte público de carga en seco y; 2) Realizar un estudio sobre la integración de competencias directivas y de su desempeño, a razón de datos cuantitativos, con el fin de minimizar la ambigüedad generada por el análisis hacia la percepción que proporciona cada directivo.

\section{Referencias}

Amaratunga, D., Baldry, D. \& Sarshar, M. (2001). Process improvement through performance measurement: the balanced scorecard methodology. Work Study, 50(5), 179-189. https://doi.org/10.1108/ EUMooooooooo5677

Beckhard, R. (1986). Desarrollo organizacional: estrategias y modelos. Fondo Educativo Interamericano.

Bell, D. (1973). The coming of post-industrial society. Basic Books, American Quarterly.

Boyatzis, R. (2002). El desarrollo de competencias sin valores es como el sexo sin amor. Journal of work and organizational psychology, 18(2-3), 247-258. https://journals.copmadrid.org/jwop/ art/301adoe3bd5cb1627a2044908a42fdc2

Blunt, A., Bennett, D., Clark, B., Taylor-Green, L. \& Singh, P. (2014). Human Resources Professional Competency Framework. Human Resources Professionals Association.

Cameron, K. S. y Quinn, R. E. (1999). Diagnosing and changing organizational culture. Base on the Competing Values Framework. Addison Wesley.

Concha, X. \& Arredondo, G. (2020). Evaluación y certificación de competencias laborales de las personas en chile: el caso de la comisión del sistema nacional de certificación de competencias laborales, Chilevalora. Cidalia. https://cutt.ly/GYeNBxJ

Cruz, E., Ortega, A., Garnica, J., Hernández, E., Robles, C. \& Castillo, O. (2018). Theoretical Foundation of a HumanTechno-Structural Model of Managerial Competences for the Public Transport of Dry Cargo. American Journal of Industrial and Business Management, 8(4), 1073-1092. https://dx.doi.org/10.4236/ ajibm.2018.84074

Cruz Aldana, E. \& Guerrero Azpeitia, L.A. (2021). Evaluación vertical y horizontal: Una integración entre el Supply Chain Operations Reference y el Balanced Scordcard. Religación. Revista De Ciencias Sociales y Humanidades, 6(27), 177-195. https://doi.org/10.46652/rgn.v6i27.746

Hernández, R., Fernández, C., \&Baptista, P. (2005). Metodología de la investigación. Mc Graw Hill.

Hammel, K.E., Hamel, G., \& Prahalad, C. K. (1995). Compitiendo por el futuro. Estrategia comercial para crear los mercados del mañana. Editorial Ariel, S.A. 
Herrera, A., Bustos, A., Martner, C., Rico, A., Acha, J., Aguerrebere, R., Heredia, F., \& Gradilla, L. (2005). Diagnóstico del transporte de carga aérea en México. Publicación Técnica No. 273. Instituto Mexicano del Transporte. http://imt.mx/archivos/Publicaciones/PublicacionTecnica/pt273.pdf

INEGl (2018). Banco de datos Directorio Estadístico Nacional de Unidades Económicas. https://www.inegi. org.mx/app/biblioteca/ficha.html?upc=702825106607

Inside Careers (2021, 18 de septiembre). Skills \& Training: Skills required for logistic \& transport. https:// www.insidecareers.co.uk/career-advice/consultancy-qualifications/

Jensen, B. (2000). Simplicity-The New Comparative Advantage. Perseus Press.

Jiménez, J.E., \& Jiménez, J. (2016). Logística del autotransporte de carga: Estrategias de gestión. Instituto Mexicano del Transporte. http://imt.mx/archivos/Publicaciones/PublicacionTecnica/pt483.pdf

Jiménez, E. (2018). Impacto del precio del diésel y otros insumos en los costos de operación del autotransporte de carga. Publicación Técnica No. 536. Instituto Mexicano del Transporte. https://www.imt.mx/archivos/ Publicaciones/PublicacionTecnica/pt536.pdf

Ortega A.O., Cruz E., \& Figueroa H. (2015). Marco contextual de un modelo humano tecno estructural de competencias directivas para el autotransporte de carga. En S. Téllez, M. Cedillo \& J. Jiménez (Ed.), Logística y cadena de suministro: retos y desafíos de México. (pp. 22-42). Universidad Politécnica de Guanajuato.

Kaplan, R., \& Norton D. (2001). Cuadro de Mando Integral. Edición Gestión 2000 S.A.

Katz, R.L. (1974). Skills of an effective Administrator. Harcar Business Review.

Levy-Leboyer, C. (1997). Evaluación del Personal: los métodos a elegir. Díaz de Santos.

McClelland, D.C. (1973). Testing for competence rather than for "intelligence". American Psychologist, 28(1), 1-14. https://psycnet.apa.org/doi/10.1037/hoo34092

Mercado, J., Del Moral, E., \& Jiménez J. (2011). Diseño de cuadro de mando integral aplicado a la integración del transporte en la cadena de 120 suministros (La quinta Perspectiva del balance scorecard). Publicación Técnica No. 347. Instituto Mexicano del Transporte. http://imt.mx/archivos/Publicaciones/ PublicacionTecnica/pt347.pdf

México Competitivo. (2021, 12 de septiembre). El International Institute for Management Development (IMD) publicó los resultados del Anuario de Competitividad Mundial 2021. https://cutt.ly/mYeBNKb

Mintzberg, H. (1973). The Nature of Managerial Work. Harper \& Row.

Morales, C.G. (2016). Evolución de la flota de autotransporte refrigerado en México (2005-2015). Publicación Técnica No. 461. Instituto Mexicano del Transporte. https:/www.imt.mx/archivos/Publicaciones/ PublicacionTecnica/pt461.pdf

Naghi, M. (2010). Metodología de la investigación. Limusa.

Olabarrieta, J.C. (1998). ¿Vino viejo en nuevo envase? Training \& Development Digest, 10, 92-95.

Porter, M. (1999). Estrategia competitiva. Técnicas para el análisis de los sectores Industriales y de competencia. C.E.C.S.A.

Prahalad, C. K., \& Bettis R. (1995). The dominant logic: Retrospective and extension. Strategic Management Journal, 16(1), 5-14. https://www.jstor.org/stable/2486943

Quinn, R.E., Faerman, S.R., Thompson, M.P., \& Mcgrath, M.R. (1990). Becoming a master manager. Wiley \& Sons.

Redwood, S., Goldwasser, C. \& Street, S. (1999). Action Management: Practical Strategias for Making your Corporate Transformation a Success. Wiley.

Rico, O. (1998). Evolución de la industria del autotransporte de carga en México en el periodo 1988-1993. Publicación Técnica No. 100. Instituto Mexicano del Transporte. https://www.imt.mx/archivos/ Publicaciones/PublicacionTecnica/pt10o.pdf 
Schein, E.H. (1978). Career Dynamics: Matching Individual and Organizational Needs. Addison-Wesley Publishing Company.

SENAI (2021, 15 de septiembre). Certificación de personas. https://cutt.ly/ZYeBIDo

Taylor, F. W. (1967). Los principios de la gerencia cientifica. Norton.

Tracy, M., \& Wierseme. F. (1995). Discipline of Market Leaders. Addison Westey Publishing Company.

Torres, Z. \& Navarro, J. (2007). Conceptos y principios fundamentales de Epistemología y de Metodología. ININEE. Universidad Michoacana de San Nicolás de Hidalgo

Tovar, E. (2011). Zonas metropolitanas en el estado de Hidalgo y cooperación intermunicipal. Argumentos. Estudios críticos de la sociedad, 24(66), 155-177. https://argumentos.xoc.uam.mx/index.php/argumentos/ article/view/292/291

Whetten, D., \& Cameron, K. (2005). Desarrollo de habilidades directivas. Pearson.

\section{AUTORES}

Eduardo Cruz Aldana. Doctor en Ciencias de Ingeniería Industrial por la UAEH, Profesor-Investigador en el Subsistema de Universidades Politécnicas desde 2010.

Luis Arturo Guerrero Azpeitia. Doctor en Ciencias de la Educación por la Universidad Autónoma del Estado de Hidalgo (UAEH), México. Actualmente es Profesor-Investigador en la Universidad Politécnica Metropolitana de Hidalgo. 